\title{
Surgical approaches to posterior polar cataract: a review
}

AR Vasavada, SM Raj, V Vasavada and S Shrivastav

\section{Introduction}

The aim of this study is to provide a comprehensive overview of surgical methods used in the emulsification of posterior polar cataracts (PPCs) that have been devised to minimize the risk of posterior capsule rupture (PCR) and its consequences. A Pubmed and Medline search of relevant literature on PPC was done. Only articles relevant to the treatment of PPC were included. The posterior capsule in eyes with PPC are known to have an abnormal adhesion to the polar opacity or a pre-existing weakness of the capsule that predisposes the eye to PCR. To circumvent the consequences of cleaving the abnormal adhesion, a majority of the surgeons use the anterior approach through the limbus, whereas some advocate the posterior approach through the pars plana. Emulsifying the nucleus and cleaving the central opacity of the PPC off the posterior capsule without disrupting its integrity provides optimal surgical outcomes. To achieve this, various modifications have been applied by surgeons during different phases of surgery. The advantages, disadvantages, complications, and results of each method have been discussed. Phacoemulsification is the preferred technique for removing PPC. This review will provide methods to avoid and /or deal with intraocular surgical difficulties that can arise during emulsification. Employing these would result in least ocular morbidity and satisfactory visual outcomes for the patient. This is particularly relevant given the major advancements in technology and refinements in surgical techniques in phacoemulsification.

Eye (2012) 26, 761-770; doi:10.1038/eye.2012.33; published online 23 March 2012

Keywords: posterior polar; hydrodissection; hydrodelineation
Posterior polar cataract (PPC) poses a unique challenge to surgeons during cataract surgery. ${ }^{1}$ Depending on the clinical presentation, PPC can be divided into three categories: (1) PPC with imminent (eyes that are at a risk of) posterior capsule dehiscence (Figure 1), (2) PPC with preexisting posterior capsule dehiscence (Figures 2a and b), (3) Spontaneous dislocation. From our clinic-based population, (unpublished data) we examined 79 patients undergoing surgery for PPC between 2009 and 2010. In this group, 77 of $79(97 \%)$ eyes displayed PPC with imminent dehiscence; 2 of 79 (3\%) eyes had PPC with pre-existing posterior capsule dehiscence. Currently, there is no surgical technique to eliminate the occurrence of posterior capsule rupture (PCR). We have reviewed suggested surgical approaches to reduce the possibility of PCR during cataract surgery of PPC with imminent posterior capsule dehiscence. We also describe surgical techniques that reduce the morbidity of the ocular tissue in eyes with pre-existing posterior capsule dehiscence and dislocated lenses.

\section{Clinical presentation}

PPC with imminent posterior capsule dehiscence

The common symptom is glare disability in photopic illumination conditions. The symptoms ameliorate if there is low illumination. Two types of PPC have been described in literature: stationary and progressive. ${ }^{1}$ Stationary PPC is characterized by a central, dense, disk-shaped opacity located on the posterior capsule with concentric rings around the central plaque opacity that appear like a bull's eye (Figure 1). The opacity has a cone-shaped projection in the subcapsular region or central posterior cortex. This type of PPC is compatible with good vision. In progressive PPC (Figure 3), changes take place
Iladevi Cataract and IOL Research Centre, Raghudeep Eye Clinic, Memnagar, Ahmedabad, India

Correspondence: AR Vasavada, lladevi Cataract and IOL Research Centre, Raghudeep Eye Clinic, Gurukul Road, Memnagar, Ahmedabad 380 052, India Tel: + 917927492303 , 27490909;

Fax: +9179 27411200

E-mail: icirc@

abhayvasavada.com

Received: 27 May 2011 Accepted in revised form: 1 November 2011 Published online: 23 March 2012 
in the posterior cortex in the form of radiating rider opacities. Patients with progressive opacity become more symptomatic as the peripheral extensions enlarge. The average age of patients that attended our clinic was 50.67 years with patients ranging from 27 to 63 years (unpublished data). Despite complaints of glare disability, the corrected visual acuity was 20/20 in these patients. It was noted that PPC was present bilaterally in $90 \%$ of the cases in our study and in $70 \%$ of cases in a study conducted by Gavris et al. ${ }^{2}$

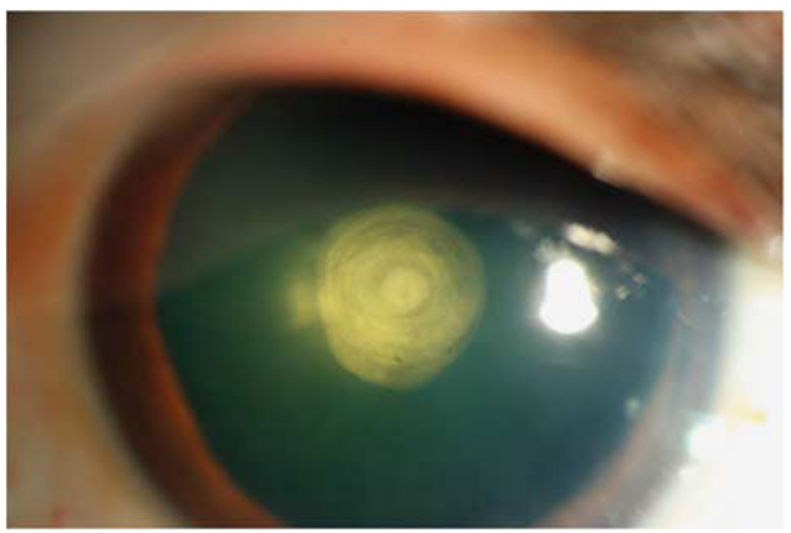

Figure 1 PPC characterized by a central dense disk-shaped opacity located on the posterior capsule with concentric rings around the central plaque opacity that appear like a bull's eye.
A suggested method to classify PPC is according to the size and density of the opacities. ${ }^{3}$ The authors denote the size of the opacity between 0.5 and $3 \mathrm{~mm}$ in increments of $0.5 \mathrm{~mm}$. The density is staged from $1+$ to $4+$ and increasing density is judged by the ability to visualize the posterior capsule.

\section{PPC with pre-existing posterior capsule dehiscence}

A posterior capsule defect is observed as an ellipticalshaped defect (Figure 2), which is generally vertically oriented with a central bull's eye-shaped opacity. It is also described as iridescent refractile lens particles in the anterior vitreous and onion skinning of the central dense plaque. ${ }^{4}$

\section{Co-existing ocular association}

A majority of the eyes (93.7\% (74 eyes of 79)) with PPC have emmetropia with axial length ranging between 21 and $24 \mathrm{~mm}$ (our unpublished data). In this data, a small proportion of patients had an axial length of $>25 \mathrm{~mm}$ and none of the eyes had an axial length of $<21 \mathrm{~mm}$. Co-existing anomalies have not been reported in literature, except for a report of retinitis pigmentosa in eyes with PPC in the Siatri and Moghini study. ${ }^{5}$ Nada et $a l^{6}$ reported two children under 3 years of age with unilateral Wilm's tumor having sporadic aniridia along with PPC.
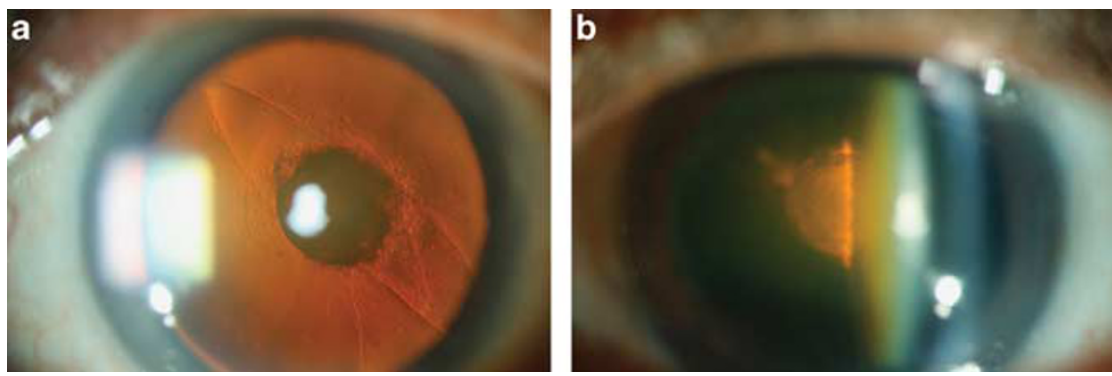

Figure 2 (a) Polar cataracts with pre-existing posterior capsule dehiscence and (b) a PPC with an intact capsule in the fellow eye.
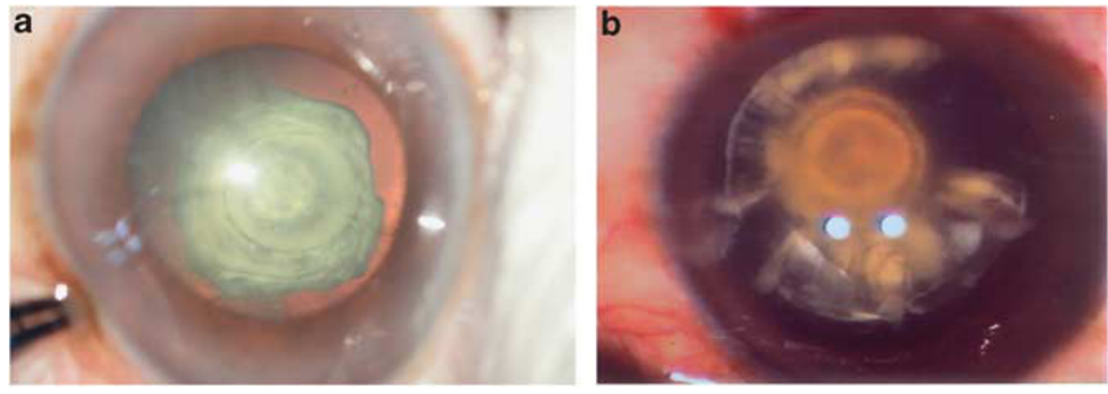

Figure $3(a, b)$ Progressive type of PPC. Changes take place in the posterior cortex in the form of radiating rider opacities. 


\section{Prognosis}

\section{Visual performance of the affected eye}

In most eyes with PPC, vision progressively worsens in bright light and while reading at close range. Visual acuity does not appear to worsen until nuclear cataract sets in. After cataract surgery, postoperative visual acuity improves to $20 / 20$ in a majority of the cases in adults.

\section{Risk of PCR during surgery}

The incidence of PCR cited in literature is as high as $36 \%$ in eyes with PPC. ${ }^{7}$ Another study reports an incidence of $26 \% .{ }^{8}$ More recently, the incidence has been reduced to 7 and $6 \% .^{9,10}$ A likely explanation for the low incidence of PCR is an understanding of the anatomical abnormality in the posterior capsule and perhaps the anterior vitreous and an understanding of the surgical factors that increase the risk of intraoperative PCR.

It was suggested that the size of the polar opacity has a significant impact on the risk of PCR. ${ }^{11}$ Overall, 7 of 23 $(30.43 \%)$ eyes with posterior polar opacities of $4 \mathrm{~mm}$ or more had PCR, whereas 2 of 35 eyes (5.71\%) with posterior polar opacities of $<4 \mathrm{~mm}$ had PCR. ${ }^{11} \mathrm{PCR}$ was found to be more common in patients $<40$ years and in the eyes that underwent extracapsular cataract extraction as compared with phacoemulslfication. ${ }^{12}$

\section{Surgical management of PPC}

Keeping in mind the potential risk of PCR during surgery, the accepted strategy would be to delay the surgery. Until the surgery is undertaken, the patient should be periodically evaluated to note visual deterioration, the opacity and integrity of the posterior capsule, and development of nuclear or cortical opacities along with PPC. If any of the above events are observed during the follow-up evaluation, the surgeon can consider surgical intervention counseling the patient regarding the risks involved during surgery and possible postoperative complications.

\section{Counseling}

During the preoperative examination, the patient is informed of the possibility of intraoperative PCR, dropped nucleus, relatively longer surgical time, secondary posterior segment intervention, and possibly delayed visual outcome. The need to perform Nd: YAG capsulotomy for residual plaque ${ }^{7-9}$ (Figure 4), and the possibility of pre-existing amblyopia especially in unilateral PPC is envisaged at the preliminary stage. ${ }^{9}$

Several surgical approaches have been proposed to minimize or delay the incidence of PCR during surgery.

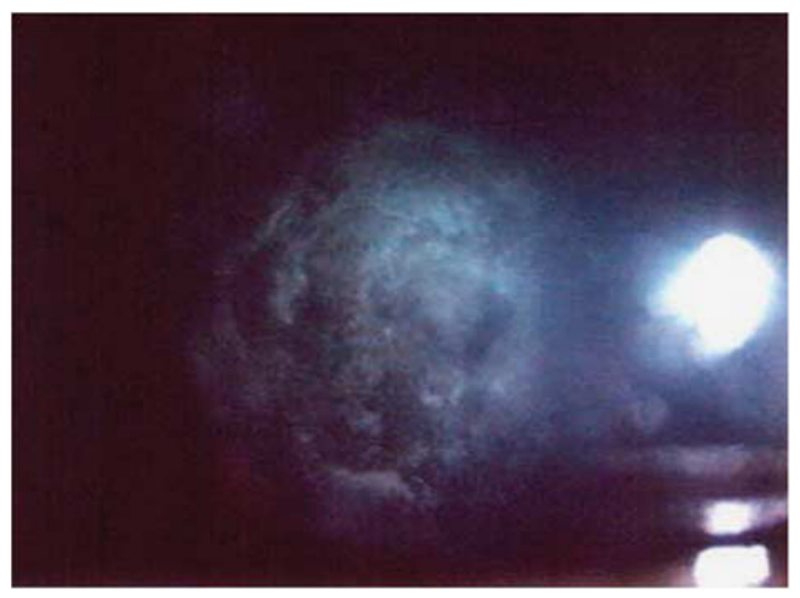

Figure 4 Residual plaque seen over an intact capsule.

Depending on the stage of PPC at the time of the presentation, the surgical approach may vary.

\section{Surgical approach in eyes with imminent posterior capsule dehiscence}

ICCE. Except for a single case, in a series of 28 eyes of 20 consecutive patients with $\mathrm{PPC},{ }^{9}$ there is no evidence for or against this approach. This approach was used by the authors in an eye with a large opacity and hard nucleus.

The posterior approach. The rationale behind using pars plana lensectomy and vitrectomy was to eliminate the risk of an unexpected capsular rupture and posterior segment complications. ${ }^{13}$ This approach was investigated in an interventional case series of 11 eyes of 8 patients. During a mean follow-up of 13 months, 3 of 11 eyes developed posterior segment complications. This approach was also used in 2 of 28 eyes undergoing surgery for PPC. The authors had used this technique as the opacity was large and the lens soft. ${ }^{9}$

The anterior approach using phacoemulsification. The goals of performing phacoemulsification in eyes with PPC are to maintain the barrier of the irido-zonular-capsular diaphragm between the anterior and posterior segments, and to implant an intraocular lens (IOL) in the bag. Surgical techniques that provide a closed chamber during all the stages of phacoemulsification can maintain the contours of the cornea and the globe. This reduces the risk of intraoperative PCR in eyes with PPC.

A number of surgical approaches have been proposed for emulsification of $\mathrm{PPC}^{14-19}$ (Table 1). We highlight the subtle variations described by authors during the different phases of this procedure (Supplementary video 1). 


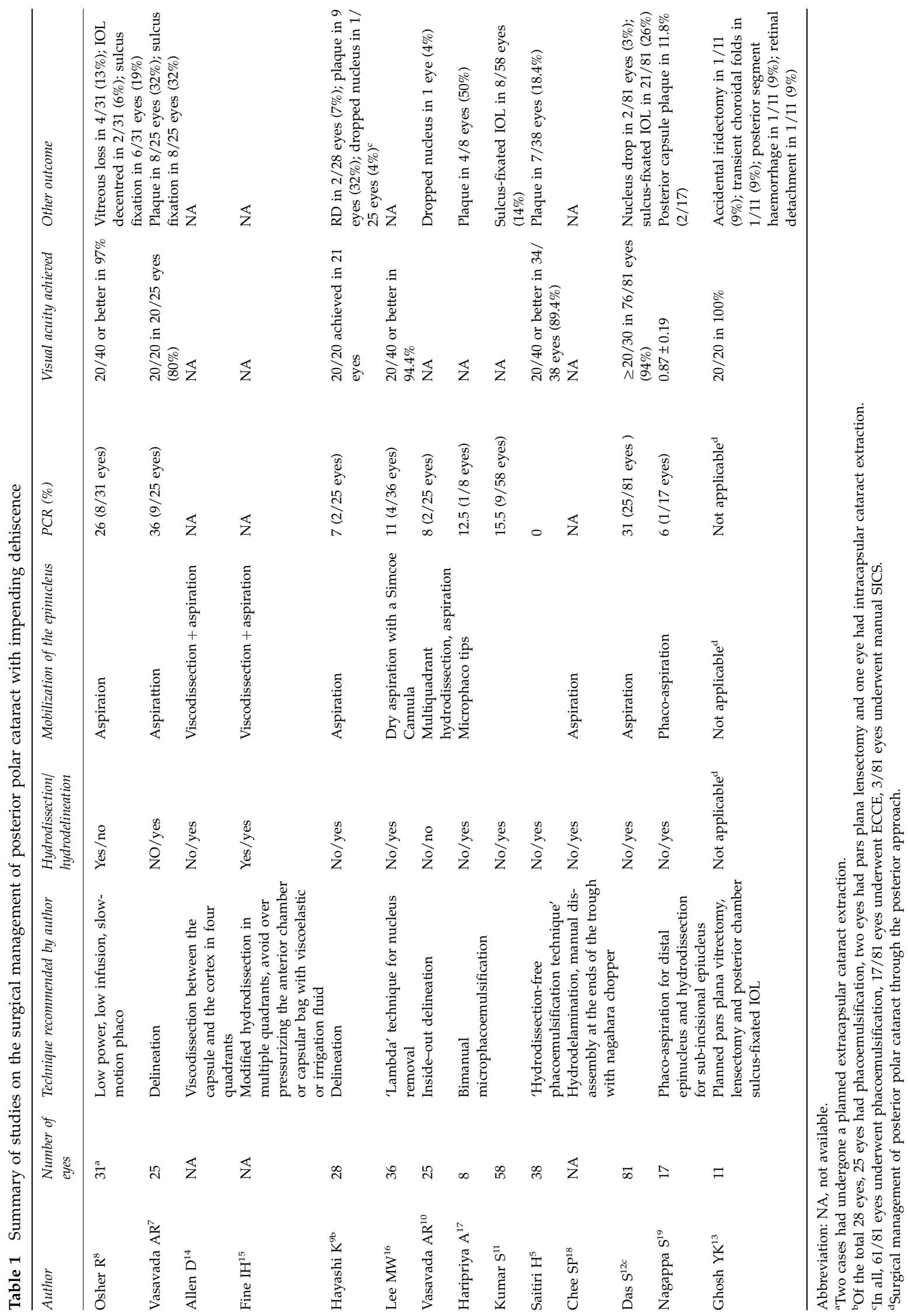


Incision. The surgery commences by creating a paracentesis incision using the $1.1 \mathrm{~mm}$ dual bevel slit knife (Alcon Surgical, Fort Worth, TX, USA). The aqueous is exchanged with Sodium Hyaluronate (Provisc, Alcon Laboratories, Fort Worth, TX, USA). A temporal, corneal, single-plane, valvular incision is created. A cohesive viscoelastic in the anterior chamber prevents chamber collapse and forward movement of the iris-lens diaphragm, during entry into the eye. Fine et $a l^{20}$ caution against increasing the pressure in the anterior chamber as this could cause a blow-out of the posterior capsule.

Capsulorhexis. The optimal size is approximately $\leq 5 \mathrm{~mm}$. A rhexis size of $\leq 4 \mathrm{~mm}$ could be detrimental in case the need arises to prolapse the nucleus into the anterior chamber. However, a larger opening may not provide adequate support for a sulcus-fixated IOL in case the posterior capsule is compromised. ${ }^{7,20}$

Hydro procedures. Cortico-cleaving hydrodissection ${ }^{15}$ can lead to hydraulic rupture and should be avoided. ${ }^{7,8}$ It would be logical to perform hydrodelineation to create a mechanical cushion of the epinucleus..$^{5,7,9,14,21}$ Masket, $^{22}$ Hayashi et al, ${ }^{9}$ Allen and Wood, ${ }^{14}$ and Lee and Lee ${ }^{16}$ recommend hydrodelineation. In addition to hydrodelineation, Fine et $a l^{20}$ also perform hydrodissection in multiple quadrants injecting tiny quantities of fluid gently, such that the fluid wave is not allowed to spread across the posterior capsule.

With conventional hydrodelineation, the cannula penetrates within the lens substance causing the fluid to traverse from outside to inside. It is sometimes difficult to introduce the cannula within a firm nucleus, as it can cause stress to the capsular bag and zonules. There is also a possibility of the fluid being injected inadvertently in the subcapsular plane, leading to unwarranted hydrodissection.

Inside-Out delineation. The authors propose 'Inside-Out Delineation' as a substitute technique that can be used instead of conventional hydro procedures as it can precisely delineate the central core of the nucleus. ${ }^{10}$ A central trench is sculpted using the slow-motion technique (Infinity Phacoemulsifier, Alcon Laboratories). Care should be taken not to mechanically rock the lens. A dispersive viscoelastic (Viscoat, Alcon Laboratories) is injected through the side-port incision before retracting the probe to avoid a forward movement of the iris-lens diaphragm. A specially designed right-angled cannula, mounted on a 2 cc syringe filled with fluid, is introduced through the main incision and the tip is placed adjacent to the right wall of the trench at an appropriate depth, depending on the density of the cataract. It then penetrates the central lens substance and fluid is injected through the right wall of the trench (Figure 5).

Delineation is produced by the fluid traversing insideout. A golden ring within the lens is evidence of successful delineation (Figure 6). Fluid injection may be repeated in the left wall of the trench with another rightangled cannula. The trench allows the surgeon to reach the central core of the nucleus (Figure 7). As fluid is injected at a desired depth, under direct vision, a desired thickness of epinucleus cushion can be achieved. It provides a precise epinucleus bowl that acts as a mechanical cushion to protect the posterior capsule during subsequent maneuvers (Figure 8). Inside-out delineation is easy to perform, provides superior control, reduces stress to the zonules, and precisely demarcates the central core of the nucleus.

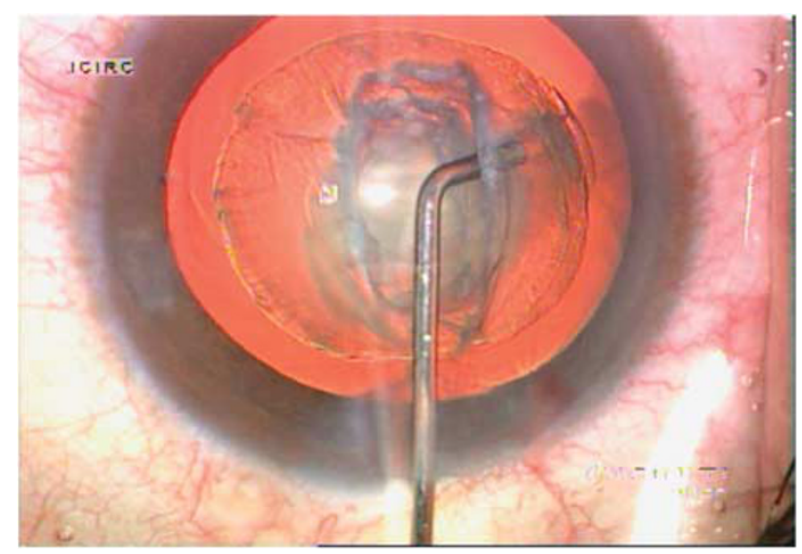

Figure 5 Demonstrates technique of inside-out delineation.

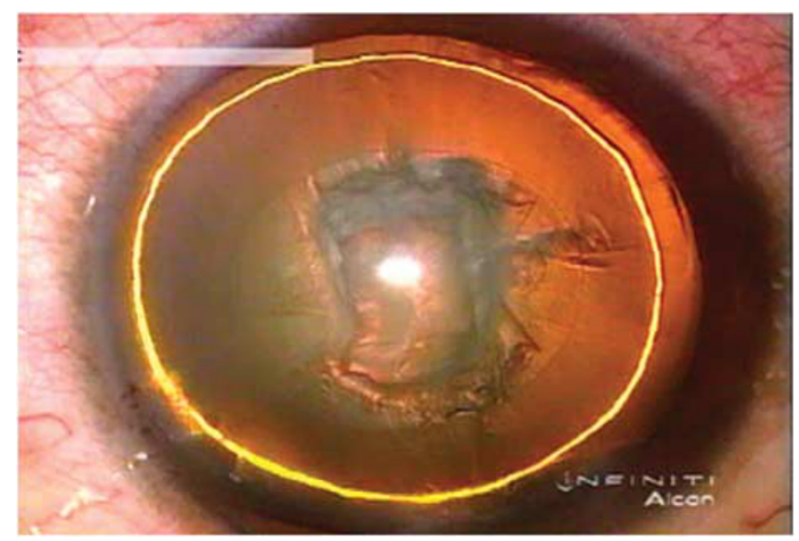

Figure 6 A golden ring within the lens is evidence of successful delineation. 


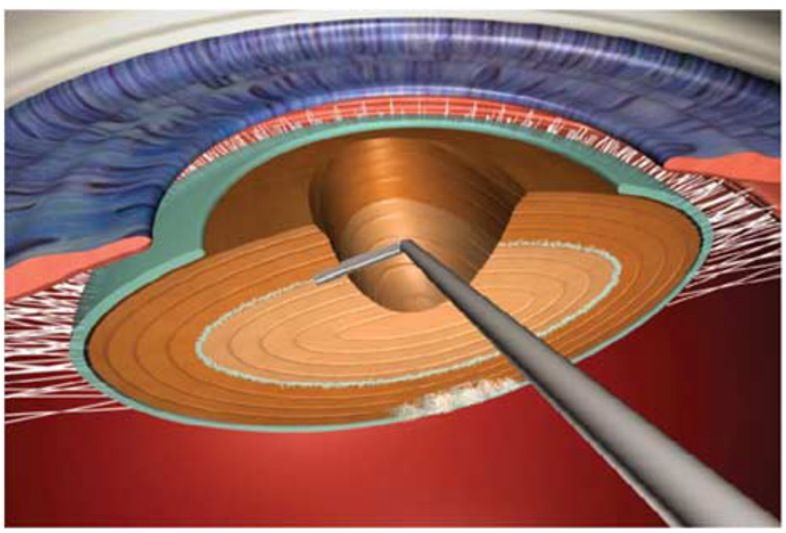

Figure 7 A sketch to demonstrate the technique of inside-out delineation.

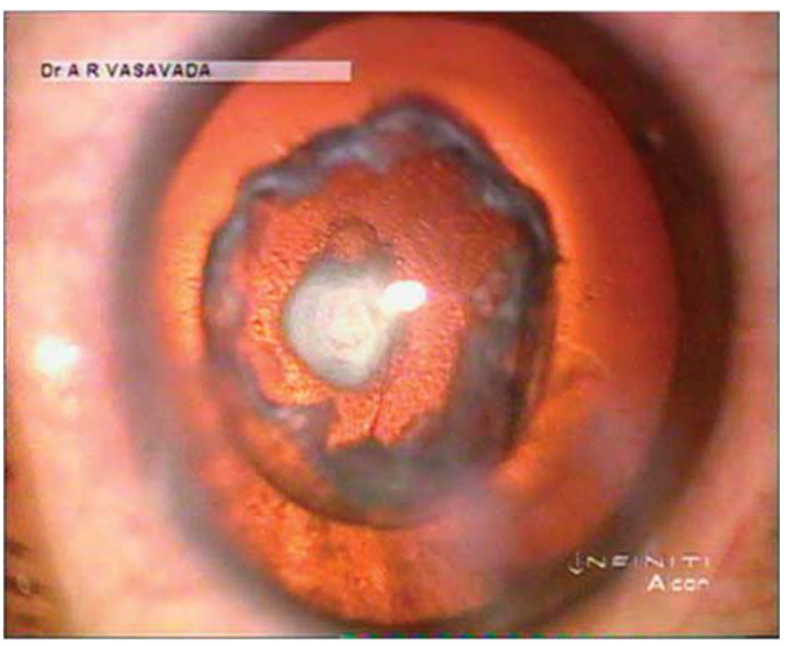

Figure 8 The residual epinucleus bowl provides mechanical protection (cushion effect) to the polar opacity during emulsification of the nucleus. This is achieved due to the precise demarcation by inside-out delineation.

Rotation. Any attempt to rotate the nucleus can lead to PCR and is best avoided. ${ }^{7}$

In dense nuclear sclerosis, Lim and $\mathrm{Goh}^{23}$ suggest prechopping the anterior epinucleus before mobilizing, segmenting, and emulsifying the dense endonucleus.

Division and fragment removal. It would be ideal to aspirate the nucleus within the cushion of the epinucleus to protect and tamponade the posterior capsule.

Bimanual cracking and division of the nucleus involve outward movements and can result in distortion of the capsular bag. In nuclear sclerosis $<2$, the demarcated nucleus is emulsified by creating adjacent trenches to create a bowl. In nuclear sclerosis of $\geq 2$, we use the step-by-step, chop in situ, and lateral separation

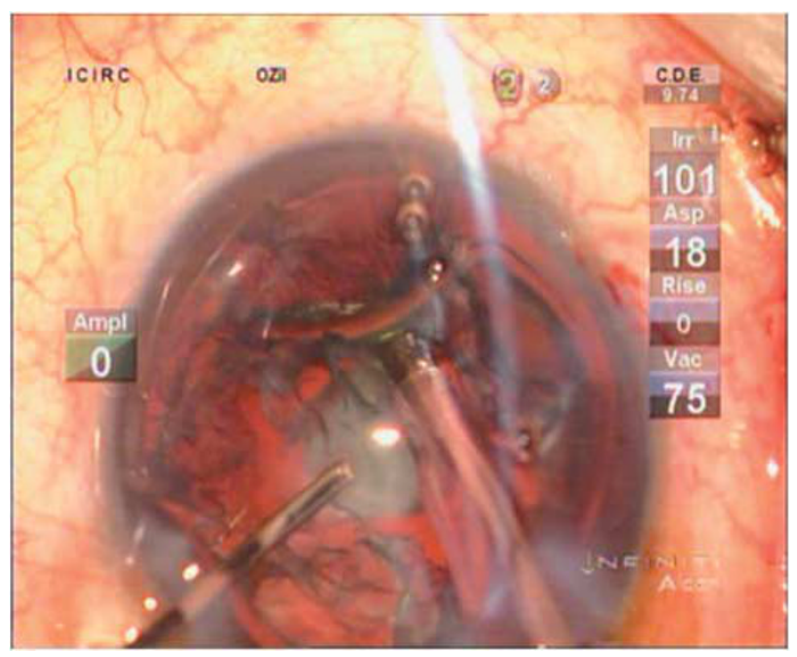

Figure 9 Cleaving the epinucleus in the clock hours opposite to the main incision from where the probe is introduced. The surgeon attempts to cleave the nasal quadrant in this illustration as the phacoemulsification is performed from the temporal approach. No attempt is made to aspirate the epinucleus at this point.

technique. ${ }^{24}$ Traction of the posterior lens fibers and posterior polar opacity during surgery are enough to break the weak posterior capsule. Thus the slow-motion technique is recommended to reduce turbulence in the anterior chamber. ${ }^{25}$ The collapse of the anterior chamber and forward bulge of the PC is prevented throughout the procedure by injecting a viscoelastic before the instrument is withdrawn. ${ }^{7}$

Lee and Lee ${ }^{16}$ use the lambda technique to sculpt the nucleus, followed by the creation of a crack along both arms, and removal of the central piece.

Epinucleus removal. Epinucleus removal is done in two steps. The first step is to cleave the epinucleus from the capsule 360 degrees circumferentially and the second step is to aspirate it. For cleaving the peripheral lower half of the epinucleus, that is, the epinucleus in the clock hours opposite the main incision from where the probe is introduced, it is stripped off the capsule with the probe, leaving the central area attached (Figure 9). ${ }^{720,22}$ During this step, the surgeon attempts to separate the epinucleus and does not attempt to aspirate the epinucleus. For cleaving the peripheral upper epinucleus (subincisional epinucleus), focal and multi-quadrant hydrodissection with a right-angled cannula facing right and left is done (Figure 10). The fluid wave travels along the cleavage formed between the capsule and the lower epinucleus (Figure 11). It does not threaten the integrity of the posterior capsule. Moreover, it is safe to hydrodissect as 


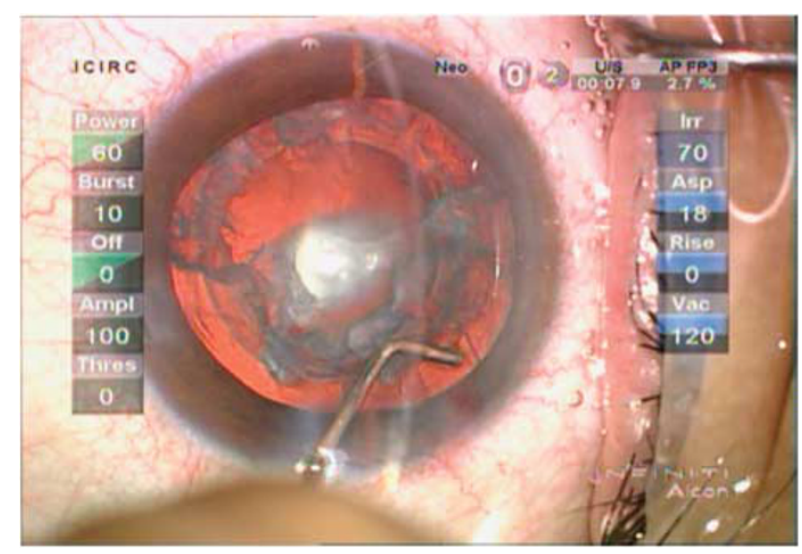

Figure 10 Cleaving the subincisional epinucleus using focal and multiquadrant hydrodissection.

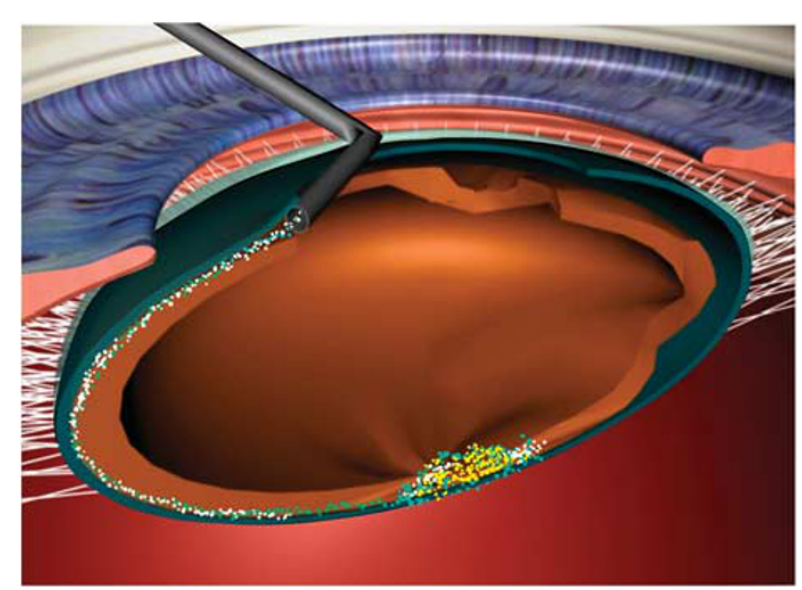

Figure 11 A sketch to demonstrate that the passage of fluid under the capsule after emulsification does not increase the hydraulic pressure within the capsular bag.

the capsular bag is not fully occupied. Therefore, the hydraulic pressure built-up is not sufficient to rupture the posterior capsule. The entire epinucleus is then aspirated, finally detaching the central area.

Allen $^{14}$ and Fine ${ }^{20}$ suggest viscodissection of the epinucleus performed by injecting a viscoelastic (Healon 5 or GV and Viscoat, respectively) under the capsular edge to mobilize the rim of the epinucleus. It is removed with a coaxial irrigation-aspiration (I/A) handpiece. Lee and Lee $^{16}$ perform manual dry aspiration with the Simcoe cannula.

Nagappa et $a l^{19}$ state that rather than just cleaving the distal epinucleus, they perform phaco-aspiration and removal of the epinucleus in the quadrant opposite to the section. Then hydrodissection is performed to release the adhesion of the sub-incisional epinucleus from the cortex. $^{19}$

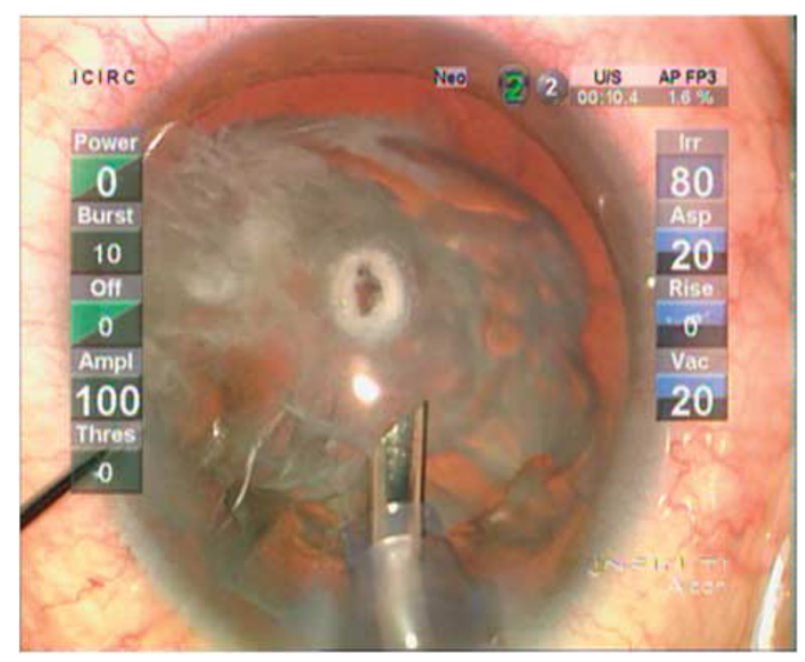

Figure 12 A pseudohole suggestive of a defect in the posterior cortex but the posterior capsule remains intact.

Highly dilute fluorescein dye has been used intracamerally to stain the epinucleus and the remaining ring-like opacity. ${ }^{26}$ The authors observe that staining the epinucleus could aid in careful cortical aspiration without causing damage to the intact posterior capsule. We speculate that in the event of an unidentified breach of the posterior capsule, injecting a dye may cause undesirable percolation into the vitreous.

Pseudohole. At times, the posterior cortex displays a classical appearance suggestive of a defect. If the posterior capsule underneath this opaque ring is intact, it is termed as a 'pseudohole' (Figure 12). Nagappa et al ${ }^{19}$ have coined the term' fish mouthing' as a sign of posterior capsular rupture where the vitreous is seen coming through the PPC.

Cortex removal. Bimanual automated I/A optimizes control, ensures anterior chamber maintenance, and aids in complete removal of the cortex. Fine et $a l^{20}$ use coaxial I/A to protect the PC with ophthalmic viscoelastic devices during cortex removal.

Posterior capsule vacuum polishing. It is avoided even if the PC is not open, because of its potential fragility. ${ }^{7-9,20,22}$ The traction on an excessively adhered plaque as compared with an otherwise normal posterior capsule could eventually rupture the posterior capsule. Instead, postoperative neodymium-yttrium-aluminum-garnet (Nd:YAG) laser posterior capsulotomy is preferable. 


\section{Surgical approach in eyes with a pre-existing defect in the posterior capsule}

The anterior approach. In an attempt to maintain an intact vitreous face in eyes, the authors follow the same paradigms as those used in eyes with an intact posterior capsule. Viscoat, which is a dispersive viscoelastic, is injected over the area of the capsule defect before the phaco or I/A probe is withdrawn from the eye..$^{27}$ The viscoat tamponades the vitreous face preventing it from prolapsing. A two-port limbal anterior vitrectomy is then performed using a maximum cut rate/min with a very low bottle height of $20-30 \mathrm{~cm}$. Once the anterior chamber is free of vitreous, the cortex is aspirated by bimanual I/A. A posterior continuous curvilinear capsulorhexis (PCCC) may be performed if the rupture is confined to a small central area. ${ }^{28}$ High-viscosity viscoelastic sodium hyaluronate is injected around the area of the initial puncture to achieve a flat or concave capsule. ${ }^{29,30}$ The ends of the tear are grasped with microforceps (ASICO, Westmont, IL, USA) and moved circumferentially to create a circular opening in the posterior capsule. The end result should be a PCCC concentric to and smaller than the anterior capsulorhexis.

Vajpayee $e t a l^{4}$ performed 'layer by layer' phacoemulsification in eight patients with pre-existing posterior capsule defects. Vitrectomy was not required in any eye and the authors found a vertical posterior capsule defect with an intact vitreous face in all eyes.

Site of IOL implantation. In eyes with a posterior capsule defect, in-the-bag IOL implantation can be considered only if PCCC is achieved. In eyes with a large posterior capsule defect, the IOL could be placed in the ciliary sulcus, provided that the lower portion of the IOL has a circumferentially uniform capsular support. Fine $e t a^{20}$ have suggested optic capture through anterior capsulorhexis. This technique was first suggested in a case of posterior capsular tear. ${ }^{31}$ The conventional or posterior optic capture can be achieved by capturing the optic through anterior capsulorhexis (haptics in the ciliary sulcus, optic in the bag). ${ }^{32}$

The technique of optic capture. A high-viscosity ophthalmic viscosurgical device, Provisc (Alcon Laboratories), is injected in the sulcus. The IOL is implanted into the sulcus. The haptics are placed in the sulcus and the optic is gently pressed backwards such that the optic gets captured in the anterior capsulorhexis. ${ }^{33}$ The anterior capsule assumes the shape of an elliptical opening. This indicates a complete optic capture. The success rate of optic capture also depends on the specific IOL design. A haptic with a right angle at the haptic-optic junction is preferred over a conventional three-piece C loop IOL design as it helps in achieving complete capsule closure.

Residual viscoelastic aspiration. After IOL implantation, the viscoelastic is removed by two-port vitrectomy rather than I/A, as vitrectomy aspirates in a piece-meal, gradual manner and reduces the chances of rapid aspiration of the vitreous.

The main valvular incision and the paracentesis should be sutured in eyes with a posterior capsule defect. In these eyes, a periodic evaluation for retinal break, cystoid macular edema, and raised intraocular pressure is necessary.

Posterior approach. On the basis of the theoretical feasibility of the pars plana approach, it is possible to perform vitrectomy and lensectomy in these eyes. However, the options for visual rehabilitation using a scleral-fixated, iris-fixated, or angle-supported lens, in circumstances where the capsular barrier can be maintained, does not seem to be a reasonable approach.

\section{Surgical approach in eyes with a spontaneous dislocation}

The clinical impression is that prolonging cataract extraction in an eye with PPC can lead to spontaneous rupture of the posterior capsule and subluxation of the lens into the vitreous. ${ }^{34,35}$ It is hypothesized that an increase in the size of the lens from nuclear sclerosis may cause increasing pressure on the posterior capsule, which subsequently ruptures because of its inherent abnormal weakness and could be a risk factor for spontaneous dislocation. ${ }^{34}$

In such eyes, a posterior approach is preferred for lensectomy via the pars plana. This can be followed by either an open-loop anterior chamber, a scleral-sutured posterior chamber, or an iris-sutured posterior chamber IOL for the correction of aphakia in eyes without adequate capsular support. ${ }^{36}$ The authors prefer scleralfixated IOLs over angle-supported IOLs. ${ }^{37,38}$

\section{PPC in children}

PPC has been identified in 7\% of eyes of children undergoing congenital cataract surgery. ${ }^{39}$ The mean age of the children in this group at the time of surgery was $6 \pm 3$ years. In another study on 33 patients aged 1 week to 8 years with lens opacities, 3 patients (9\%) had PPCs. ${ }^{40}$ Unlike adult eyes, PPC occurs as unilateral cataract in a majority of pediatric eyes $(93 \%) \cdot{ }^{39}$ Grading the severity of infantile cataracts has been proposed as a clinical guide 
to decide surgical intervention. ${ }^{40}$ Using this criteria, PPC with a grade of 6 was considered for surgical removal.

The pre-existing defect seen in eyes of children with congenital cataract is a different entity and does not appear to be a manifestation of an association with polar cataract. In our case series of 400 eyes that underwent cataract surgery for congenital cataract, a defect was present in 27 eyes (7\%). ${ }^{41}$ The preoperative diagnostic signs of a pre-existing posterior capsule defect in children include a well-demarcated defect with thick margins, chalky white spots in a cluster or a rough circle on the posterior capsule, and white dots in the anterior vitreous that move with the degenerated vitreous like a fish tail sign (Figure 13). ${ }^{41-45}$

The surgical paradigms for cataract surgery in eyes of children with PPC remain essentially similar to those used in adult eyes. ${ }^{41}$ The authors prefer to adopt the closed chamber technique. The anterior chamber is first entered using a paracentesis incision. It is then filled with a high-viscosity $1.4 \%$ sodium hyaluronate. A clear corneal incision is made. An anterior capsulorhexis is initiated by making a nick with a 26-gauge cystotome, and thereafter completed using micro-forceps by repeatedly grasping the flap. No hydro procedures are performed. This is followed by bimanual I/A using a low bottle height of $40 \mathrm{~cm}$ initially. The height is gradually increased to $70-80 \mathrm{~cm}$. If a thin posterior capsule with well-demarcated margins or a capsular flutter is noticed, posterior capsulectomy and vitrectomy are performed with a vitrector. While carrying out vitrectomy, the goal is to remove only the central anterior vitreous without attempting to remove the peripheral or posterior vitreous. This kind of limited vitrectomy is performed

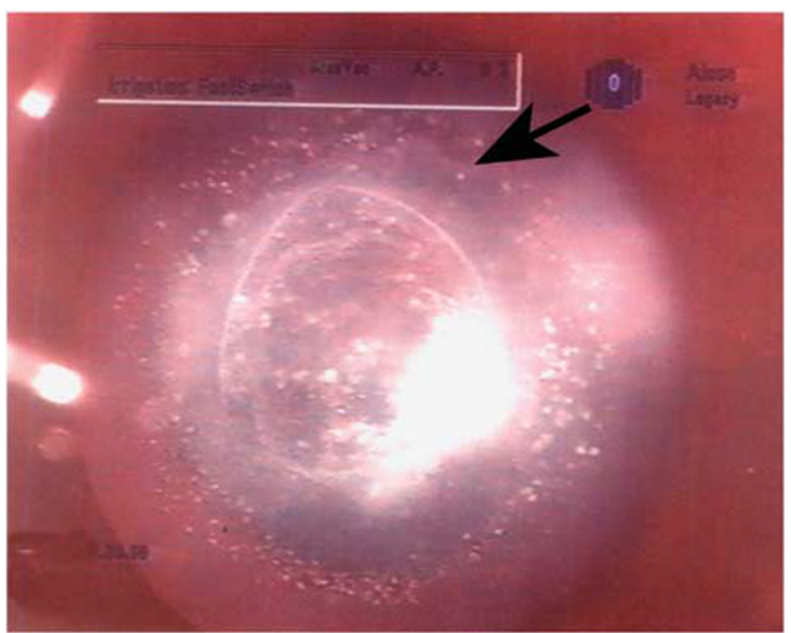

Figure 13 The preoperative diagnostic signs of a pre-existing posterior capsule defect in children. typically through two limbal (corneal) ports. During this procedure, irrigation is separated from the cutting and aspiration. This is followed by in-the-bag implantation of the AcrySof IOL (Alcon Laboratories). All the incisions are sutured.

In eyes with PPC, an improvement in visual acuity (20/40 or better) has been noted in $84 \%$ of children after surgery. ${ }^{39}$

\section{Conclusion}

PPC can pose a surgical challenge to novice surgeons. The surgical steps include performing inside-out delineation, adhering to the closed-chamber technique, adopting the slow-motion technique for lens removal, and using focal and multiquadrant hydrodissection after nucleus removal to achieve adequate cleavage of the subincisional epinucleus from the capsule. In the event of a breach in the posterior capsule in these eyes, a periodic evaluation for retinal break, cystoid macular edema, and raised intraocular pressure is necessary.

\section{Method of literature search}

For this review, we conducted a Medline and Pubmed search of medical literature using the following key words: PPC, pre-existing defect, cataract surgery, and optic capture. Reference lists from the selected articles were used to obtain further relevant articles not included in the electronic database. Only articles pertinent to the surgical approaches used for PPC were included.

\section{Conflict of interest}

The authors declare no conflict of interest.

\section{References}

1 Duke-Elder S. Posterior polar cataract. In: Duke-Elder S (ed). System of Ophthalmology 3, Pt 2 Normal and Abnormal Development, Congenital Deformities. CV Mosby: St Louis, MO, 1964, pp 723-726.

2 Gavris M, Popa D, Caraus C, Gusho E, Clocotan D, Horvath $\mathrm{K}$ et al. Phacoemulsification in posterior polar cataract. Oftalmologia 2004; 48: 36-40.

3 Plechaty G, Roy H. Posterior polar cataract. Medscape reference. Available at: http://emedicine.medscape.com/ article/1211609.

4 Vajpayee RB, Sinha R, Singhvi A, Sharma N, Titiyal JS, Tandon R. 'Layer by layer' phacoemulsification in posterior polar cataract with pre-existing posterior capsular rent. Eye 2008; 22: 1008-1010.

5 Siatiri H, Moghimi S. Posterior polar cataract: minimizing risk of posterior capsule rupture. Eye 2006; 20: 814-816.

6 Nada M, Rattan KN, Magu S, Parshad S. Aniridia and Wilm's tumor. Indian J Pediatr 2003; 70: 837-838. 
7 Vasavada AR, Singh R. Phacoemulsification with posterior polar cataract. J Cataract Refract Surg 1999; 25: 238-245.

8 Osher RH, Yu BC, Koch DD. Posterior polar cataracts: a predisposition to intraoperative posterior capsular rupture. J Cataract Refract Surg 1990; 16: 157-162.

9 Hayashi K, Hayashi H, Nakao F et al. Outcomes of surgery for posterior polar cataract. J Cataract Refract Surg 2003; 29: $45-49$.

10 Vasavada AR, Raj SM. Inside-out delineation. J Cataract Refract Surg 2004; 30: 1167-1169.

11 Kumar S, Ram J, Sukhija J, Severia S. Phacoemulsification in posterior polar cataract: does size of lens opacity affect surgical outcome? Clin Experiment Ophthalmol 2010; 38: 857-861.

12 Das S, Khanna R, Mohiuddin SM, Ramamurthy B. Surgical and visual outcomes for posterior polar cataract. $\mathrm{Br} J$ Ophthalmol 2008; 92: 1476-1478.

13 Ghosh YK, Kirkby GR. Posterior polar cataract surgery - a posterior segment approach. Eye 2008; 22: 844-848.

14 Allen D, Wood C. Minimizing risk to the capsule during surgery for posterior polar cataract. J Cataract Refract Surg 2002; 28: 742-744.

15 Fine IH. Cortico-cleaving hydrodissection. J Cataract Refract Surg 1992; 18: 508-512.

16 Lee MW, Lee YC. Phacoemulsification of posterior polar cataracts- a surgical challenge. Br J Ophthalmol 2003; 87: 1426-1427.

17 Haripriya A, Aravind S, Vadi K, Natchiar G. Bimanual microphaco for posterior polar cataracts. J Cataract Refract Surg 2006; 32: 914-917.

18 Chee SP. Management of the hard posterior polar cataract. Cataract Refract Surg 2007; 33: 1509-1514.

19 Nagappa S, Das S, Kurian M, Braganza A, Shetty R, Shetty B. Modified technique for epinucleus removal in posterior polar cataract. Ophthalmic Surg Lasers Imaging 2011; 42: 78-80.

20 Fine IH, Packer M, Hoffman RS. Management of posterior polar cataract. J Cataract Refract Surg 2003; 29: 16-19.

21 Aziz YA. Understanding hydrodelineation: The term and procedure. Doc Ophthalmol 1994; 87: 123-137.

22 Masket S. Consultation Section. J Cataract Refract Surg 1997; 23: 819-824.

23 Lim Z, Goh J. Modified epinucleus pre-chop for the dense posterior polar cataract. Ophthalmic Surg Lasers Imaging 2008; 39: 171-173.

24 Vasavada AR, Singh R. Step-by-step chop in situ and separation of very dense cataracts. J Cataract Refract Surg 1998; 24: 156-159.

25 Osher RH, Cionni R. In: Steinert RF (2nd edn). Cataract Surgery, Technique, Complications, Management. Saunders: Philadelphia, 2004, pp 469-486.

26 Hoffer KJ, McFarland JE. Intracameral subcapsular fluorescein staining for improved visualization during capsulorhexis in mature cataracts. J Cataract Refract Surg 1993; 19: 566.

27 Gimbel HV. Posterior capsule tears using phacoemulsification: causes, prevention and management. Eur J Implant Refract Surg 1990; 2: 63-69.

28 Gimbel HV. Posterior continuous curvilinear capsulorhexis and optic capture of the intraocular lens to prevent secondary opacification in pediatric cataract surgery. J Cataract Refract Surg 1997; 23(Suppl 1): 652-656.

29 Dholakia SA, Praveen MR, Vasavada AR, Nihalani B. Completion rate of primary posterior continuous curvilinear capsulorhexis and vitreous disturbance during congenital cataract surgery. J AAPOS 2006; 10: 351-356.

30 Praveen MR, Vasavada AR, Koul A, Trivedi RH, Vasavada VA, Vasavada VA. Subtle signs of anterior vitreous face disturbance during posterior capsulorhexis in pediatric cataract surgery. J Cataract Refract Surg 2008; 34: 163-167.

31 Neuhann T, Neuhann Th. The Rhexis-Fixated Lens,' film presented at the Symposium on Cataract IOL and Refractive Surgery, Boston, MA, USA, 1991.

32 Gimbel HV, DeBroff BM. Posterior capsulorhexis with optic capture: maintaining a clear visual axis after pediatric cataract surgery. J Cataract Refract Surg 1994; 20: 658-664.

33 Vasavada AR, Trivedi R. Role of optic capture in congenital cataract and IOL surgery in children. J Cataract Refract Surg 2000; 26: 824-831.

34 Ho SF, Ahmed S, Zaman AG. Spontaneous dislocation of posterior polar cataract. J Cataract Refract Surg 2007; 33: 1471-1473.

35 Ashraf H, Khalili MR, Salouti R. Bilateral spontaneous rupture of posterior capsule in posterior polar cataract. Clin Experiment Ophthalmol 2008; 36: 798-800.

36 Wagoner MD, Cox TA, Ariyasu RG, Jacobs DS, Karp CL. American Academy of Ophthalmology. Intraocular lens implantation in the absence of capsular support: a report by the American Academy of Ophthalmology. Ophthalmology 2003; 110: 840-859. Review.

37 Monteiro M, Marinho A, Borges S, Ribeiro L, Correia C. Scleral fixation in eyes with loss of capsule or zonule support. J Cataract Refract Surg 2007; 33: 573-576.

38 Hara T, Hara T. Ten-year results of anterior chamber fixation of the posterior chamber intraocular lens. Arch Ophthalmol 2004; 122: 1112-1116.

39 Mistr SK, Trivedi RH, Wilson ME. Preoperative considerations and outcomes of primary intraocular lens implantation in children with posterior polar and posterior lentiglobus cataract. J AAPOS 2008; 12: 58-61.

40 Forster JE, Abadi RV, Muldoon M, Lloyd IC. Grading infantile cataracts. Ophthalmic Physiol 2006; 26: 372-379.

41 Vasavada AR, Praveen MR, Nath V, Dave K. Diagnosis and management of congenital cataract with preexisting posterior capsule defect. J Cataract Refract Surg 2004; 30: 403-408.

42 Singh D, Singh R, Singh I. Cataract and IOL. Jaypee Brothers: New Delhi, 1993, pp 160-167.

43 Vajpayee RB, Angra SK, Honavar SG, Titiyal JS, Sharma YR, Sakhuja N. Pre-existing posterior capsule breaks from perforating ocular injuries. J Cataract Refract Surg 1994; 20: 291-294.

44 Vasavada AR, Praveen MR, Dholakia SA, Trivedi RH. Preexisting posterior capsule defect progressing to white mature cataract. J AAPOS 2007; 11: 192-194.

45 Vasavada AR, Praveen MR, Tassignon MJ, Shah SK, Vasavada VA, Vasavada VA et al. Posterior capsule management in congenital cataract surgery. J Cataract Refract Surg 2011; 37: 173-193. Review.

Supplementary Information accompanies the paper on Eye website (http://www.nature.com/eye) 